PROCEEDINGS OF THE

AMERICAN MATHEMATICAL SOCIETY

Volume 135, Number 4, April 2007, Pages 1081-1088

S 0002-9939(06)08612-6

Article electronically published on October 2, 2006

\title{
PRINCIPLE OF LOCAL REFLEXIVITY REVISITED
}

\author{
EVE OJA AND MÄRT PÕLDVERE
}

(Communicated by N. Tomczak-Jaegermann)

\begin{abstract}
We give, departing from Grothendieck's description of the dual of the space of weak*-weak continuous finite-rank operators, a clear proof for the principle of local reflexivity in a general form.
\end{abstract}

\section{Introduction and the Main Lemma}

Let $X$ and $Y$ be Banach spaces (both over $\mathbb{K}=\mathbb{R}$ or $\mathbb{C}$ ). The closed unit ball of $X$ is denoted by $B_{X}$, its unit sphere by $S_{X}$, and the identity operator on $X$ by $I_{X}$. We shall consider $X$ as a subspace of $X^{* *}$, identifying the canonical embedding $j_{X}: X \rightarrow X^{* *}$ with the identity embedding. We denote by $\mathcal{L}(X, Y)$ the Banach space of bounded linear operators from $X$ to $Y$, and by $\mathcal{F}(X, Y)$ its subspace of finite-rank operators. The subspace of $\mathcal{L}\left(X^{*}, Y\right)$ consisting of weak*weak continuous finite-rank operators is denoted by $\mathcal{F}_{w^{*}}\left(X^{*}, Y\right)$.

Recall that any $T \in \mathcal{F}_{w^{*}}\left(X^{*}, Y\right)$ can be written as $T=\sum_{i=1}^{n} x_{i} \otimes y_{i}$ for some $x_{i} \in X$ and $y_{i} \in Y$, where the operator $x_{i} \otimes y_{i} \in \mathcal{F}_{w^{*}}\left(X^{*}, Y\right)$ is defined by $\left(x_{i} \otimes y_{i}\right) x^{*}=x^{*}\left(x_{i}\right) y_{i}, x^{*} \in X^{*}$. Note that then $T^{*}=\sum_{i=1}^{n} y_{i} \otimes x_{i}$. By a well-known description, due to Grothendieck [G, Chapter I, pages 124-125] (see, e.g., [DU, pages 231-232] or [ $\underline{\mathrm{R}}$, page 58]), $\mathcal{F}_{w^{*}}\left(X^{*}, Y\right)^{*}=\mathcal{I}\left(X, Y^{*}\right)$, the Banach space of integral operators (equipped with their integral norms). This identification $\mathcal{F}_{w^{*}}\left(X^{*}, Y\right)^{*}=\mathcal{I}\left(X, Y^{*}\right)$ is realized via the duality

$$
\left\langle A, \sum_{i=1}^{n} x_{i} \otimes y_{i}\right\rangle=\sum_{i=1}^{n}\left(A x_{i}\right)\left(y_{i}\right) .
$$

In particular, for all $x^{*} \otimes y^{*} \in \mathcal{F}_{w^{*}}\left(X^{*}, Y\right)^{*}, x^{*} \in X^{*}, y^{*} \in Y^{*}$, and $T \in$ $\mathcal{F}_{w^{*}}\left(X^{*}, Y\right)$, one has $\left\langle x^{*} \otimes y^{*}, T\right\rangle=x^{*}\left(T^{*} y^{*}\right)$.

Lemma 1.1 (Main Lemma). Let $X$ and $Y$ be Banach spaces. If $T \in \mathcal{F}_{w^{*}}\left(X^{*}, Y^{* *}\right)$, then there exists a net $\left(T_{\alpha}\right) \subset \mathcal{F}_{w^{*}}\left(X^{*}, Y\right)$ satisfying

$1^{\circ}\left\|T_{\alpha}\right\| \rightarrow\|T\|$,

$2^{\circ} T_{\alpha} x^{*} \rightarrow T x^{*}$ for all $x^{*} \in T^{-1}(Y)$,

$3^{\circ} T_{\alpha}^{*} y^{*} \rightarrow T^{*} y^{*}$ for all $y^{*} \in Y^{*}$.

Received by the editors April 6, 2005 and, in revised form, November 2, 2005.

2000 Mathematics Subject Classification. Primary 46B07, 46B20, 46B28.

Key words and phrases. Principle of local reflexivity, extension operator, locally complemented subspaces.

This research was partially supported by Estonian Science Foundation Grant 5704 .

(C)2006 American Mathematical Society 
Proof. Using the canonical identifications

$$
\mathcal{F}_{w^{*}}\left(X^{*}, Y\right)^{*}=\mathcal{I}\left(X, Y^{*}\right) \text { and } \mathcal{F}_{w^{*}}\left(X^{*}, Y^{* *}\right)^{*}=\mathcal{I}\left(X, Y^{* * *}\right),
$$

let us define $\Phi: \mathcal{F}_{w^{*}}\left(X^{*}, Y\right)^{*} \rightarrow \mathcal{F}_{w^{*}}\left(X^{*}, Y^{* *}\right)^{*}$ by $\Phi(A)=j_{Y^{*}} A, A \in \mathcal{I}\left(X, Y^{*}\right)$. Then clearly $\|\Phi\|=1$ and thus $\Phi^{*}(T) \in\|T\| B_{\mathcal{F}_{w^{*}}\left(X^{*}, Y\right)^{* *}}$. By Goldstine's theorem, there is a net $\left(T_{\alpha}\right) \subset \mathcal{F}_{w^{*}}\left(X^{*}, Y\right)$ converging weak ${ }^{*}$ to $\Phi^{*}(T)$ such that $\sup _{\alpha}\left\|T_{\alpha}\right\| \leq$ $\|T\|$. In particular, for all $x^{*} \in X^{*}$ and $y^{*} \in Y^{*}$,

$$
\begin{aligned}
x^{*}\left(T_{\alpha}^{*} y^{*}\right) & =\left\langle x^{*} \otimes y^{*}, T_{\alpha}\right\rangle \rightarrow\left(\Phi^{*}(T)\right)\left(x^{*} \otimes y^{*}\right)=\left(\Phi\left(x^{*} \otimes y^{*}\right)\right)(T) \\
& =\left\langle x^{*} \otimes j_{Y^{*}} y^{*}, T\right\rangle=x^{*}\left(T^{*} y^{*}\right) .
\end{aligned}
$$

This means that $\left.T_{\alpha}^{*} \rightarrow T^{*}\right|_{Y^{*}}$ in the weak operator topology of $\mathcal{L}\left(Y^{*}, X\right)$. Since the weak and strong operator topologies yield the same dual space (see, e.g., DSch, Theorem VI.1.4]), after passing to convex combinations, we may assume that $T_{\alpha}^{*} y^{*} \rightarrow T^{*} y^{*}$ for all $y^{*} \in Y^{*}$. This implies that $T_{\alpha} \rightarrow T$ in the weak operator topology of $\mathcal{L}\left(T^{-1}(Y), Y\right)$. Therefore, after passing to new convex combinations, we may also assume that $T_{\alpha} x^{*} \rightarrow T x^{*}$ for all $x^{*} \in T^{-1}(Y)$. Thus we have $3^{\circ}$ and $2^{\circ}$. Since $\lim \sup _{\alpha}\left\|T_{\alpha}\right\| \leq\|T\|$ and, by $3^{\circ}$,

$$
\left\|T^{*} y^{*}\right\|=\liminf _{\alpha}\left\|T_{\alpha}^{*} y^{*}\right\| \leq \liminf _{\alpha}\left\|T_{\alpha}^{*}\right\|\left\|y^{*}\right\| \quad \forall y^{*} \in Y^{*},
$$

implying that $\|T\| \leq \liminf _{\alpha}\left\|T_{\alpha}\right\|$, we also have $1^{\circ}$.

Next we show that, by a simple perturbation argument, the convergence in $2^{\circ}$ and $3^{\circ}$ can be made constant on $T^{-1}(Y)$ and on finite-dimensional subspaces of $Y^{*}$.

Lemma 1.2 (Main Lemma "fixing points"). Let $X$ and $Y$ be Banach spaces, and let $F \subset Y^{*}$ be a finite-dimensional subspace. If $T \in \mathcal{F}_{w^{*}}\left(X^{*}, Y^{* *}\right)$, then there exists a net $\left(T_{\alpha}\right) \subset \mathcal{F}_{w^{*}}\left(X^{*}, Y\right)$ satisfying $1^{\circ}, 3^{\circ}$, and

$2^{\circ \circ} T_{\alpha} x^{*}=T x^{*}$ for all $x^{*} \in T^{-1}(Y)$,

$3^{\circ \circ} T_{\alpha}^{*} y^{*}=T^{*} y^{*}$ for all $y^{*} \in F$.

Proof. (a) We first prove Lemma 1.2 in the particular case when $X$ is finitedimensional. Then $T^{-1}(Y)$ is of finite dimension.

Let $x_{1}^{*}, \ldots, x_{m}^{*} \in X^{*}$ and $y_{1}^{*}, \ldots, y_{n}^{*} \in Y^{*}$ be bases of $T^{-1}(Y)$ and $F$, respectively. Choose $x_{1}, \ldots, x_{m} \in X$ and $y_{1}, \ldots, y_{n} \in Y$ to form biorthogonal systems. Then $P=\sum_{i=1}^{m} x_{i} \otimes x_{i}^{*} \in \mathcal{F}_{w^{*}}\left(X^{*}, X^{*}\right)$ and $Q=\sum_{i=1}^{n} y_{i} \otimes y_{i}^{*} \in \mathcal{F}_{w^{*}}\left(Y^{*}, Y^{*}\right)$ are projections with $\operatorname{ran} P=T^{-1}(Y), \operatorname{ran} Q=F$, and $\operatorname{ran} Q^{*} \subset Y$.

Let $\left(T_{\alpha}\right)$ be the net in Lemma 1.1. We apply to all operators $T_{\alpha}$ the following perturbation argument, which has been inspired by the classical one due to Johnson, Rosenthal, and Zippin (see [JRZ, Lemma 2.4] or, e.g., [C, Lemma 3.2]). Namely, we define, for all $\alpha$,

$$
S_{\alpha}=T P+T_{\alpha}(I-P)-Q^{*}\left(T_{\alpha}-T\right)(I-P)=T+\left(I-Q^{*}\right)\left(T_{\alpha}-T\right)(I-P) .
$$

Then $S_{\alpha} \in \mathcal{F}_{w^{*}}\left(X^{*}, Y\right)$, because $\operatorname{ran} T P \subset Y$ and $\operatorname{ran} Q^{*} \subset Y$, and $P, T_{\alpha}$ and $T$ are weak*-weak continuous finite-rank operators. We have $S_{\alpha} x^{*}=T x^{*}$ for all $x^{*} \in T^{-1}(Y)$ because $T^{-1}(Y)=\operatorname{ran} P$ and $P^{2}=P$. Since

$$
S_{\alpha}^{*}=T^{*}+\left(I-P^{*}\right)\left(T_{\alpha}^{*}-T^{*}\right)(I-Q),
$$

$F=\operatorname{ran} Q$, and $Q^{2}=Q$, we also have $S_{\alpha}^{*} y^{*}=T^{*} y^{*}$ for all $y^{*} \in F$. 
Finally, observing that $S_{\alpha}-T_{\alpha}=\left(T-T_{\alpha}\right) P-Q^{*}\left(T_{\alpha}-T\right)(I-P)$, we have

$$
\begin{aligned}
\left\|S_{\alpha}-T_{\alpha}\right\| & \leq\left\|\left(T-T_{\alpha}\right) P\right\|+\|I-P\|\left\|\left(T_{\alpha}^{*}-T^{*}\right) Q\right\| \\
& \leq \sup _{\substack{x^{*} \in T-1 \\
\left\|x^{*}\right\| \leq\|P\|}}\left\|\left(T-T_{\alpha}\right) x^{*}\right\|+\|I-P\| \sup _{\substack{y^{*} \in F \\
\left\|y^{*}\right\| \leq\|Q\|}}\left\|\left(T_{\alpha}^{*}-T^{*}\right) y^{*}\right\| \rightarrow 0
\end{aligned}
$$

because the convergences in $2^{\circ}$ and $3^{\circ}$ are uniform on compact subsets (recall that $T^{-1}(Y)$ and $F$ are finite-dimensional spaces). This carries the properties $1^{\circ}$ and $3^{\circ}$ from $\left(T_{\alpha}\right)$ to $\left(S_{\alpha}\right)$. It only remains to relabel the net $\left(S_{\alpha}\right)$ to become $\left(T_{\alpha}\right)$.

(b) In the general case, let us factorize $T$ as $T=\tilde{T} \kappa$ where $\kappa: X^{*} \rightarrow X^{*} / \operatorname{ker} T$ is the quotient mapping and $\tilde{T}: X^{*} / \operatorname{ker} T \rightarrow Y^{* *}$ is the canonical injection. Since $\operatorname{dim}\left(X^{*} / \operatorname{ker} T\right)<\infty$, we can apply Part (a) to obtain $\left(\tilde{T}_{\alpha}\right) \subset \mathcal{F}\left(X^{*} / \operatorname{ker} T, Y\right)$ with the required properties. The net $\left(T_{\alpha}\right)$ with $T_{\alpha}:=\tilde{T}_{\alpha} \kappa$ has the same properties. Indeed, it suffices to observe (for $2^{\circ \circ}$ ) that $x^{*} \in T^{-1}(Y)$ implies $\kappa x^{*} \in \tilde{T}^{-1}(Y)$, (for $3^{\circ}$ and $3^{\circ \circ}$ ) that $T_{\alpha}^{*} y^{*}=\kappa^{*} \tilde{T}_{\alpha}^{*} y^{*}$ whenever $y^{*} \in Y^{*}$, and $\left(\right.$ for $1^{\circ}$ ) that $\|A \kappa\|=\|A\|$ whenever $A$ is a bounded linear operator defined on $X^{*} / \operatorname{ker} T$.

The principle of local reflexivity (PLR) is due to Lindenstrauss and Rosenthal [LR]. The Lindenstrauss-Rosenthal lemma was improved by Johnson, Rosenthal, and Zippin [JRZ] (see Theorem 2.3 below). Nowadays, this is the most well-known and widely used form of the PLR. Many proofs of it have been presented in the literature (see, e.g., $\mathrm{Ber}, \mathrm{D}, \overline{\mathrm{M}-\mathrm{A}},[\mathrm{S}]$ ), but they hardly "make evident why things work" (we quote Castillo Ca here). The Main Lemma suggests a unified approach for obtaining different versions of the PLR. Choosing appropriate $X, Y$, $F$ and $T \in \mathcal{F}_{w^{*}}\left(X^{*}, Y^{* *}\right)$, and letting $\left(T_{\alpha}\right)$ be the net in Lemma 1.2, the local reflexivity mapping one is looking for will be $T_{\alpha}$ with $\alpha$ "sufficiently large" (or a convex combination of such $T_{\alpha}$ ).

\section{Applications}

1. We first point out the special case of Lemma 1.2 when $E \subset Y^{* *}$ is a finitedimensional subspace, and $T \in \mathcal{F}_{w^{*}}\left(E, Y^{* *}\right)=\mathcal{F}\left(E, Y^{* *}\right)$ is the identity embedding.

Corollary 2.1. Let $Y$ be a Banach space, and let $E \subset Y^{* *}$ and $F \subset Y^{*}$ be finitedimensional subspaces. Then there exists a net $\left(T_{\alpha}\right) \subset \mathcal{F}(E, Y)$ such that

$1^{\circ}\left\|T_{\alpha}\right\| \rightarrow 1$,

$2^{\circ} T_{\alpha} y=y$ for all $y \in E \cap Y$,

$\left.3^{\circ} T_{\alpha}^{*} y^{*} \rightarrow y^{*}\right|_{E}$ for all $y^{*} \in Y^{*}$, in particular, $T_{\alpha}^{*} y^{*}=\left.y^{*}\right|_{E}$ whenever $y^{*} \in F$.

Given any $\varepsilon>0$, we can guarantee in Corollary 2.1 , by enlarging $F$ if necessary, that the operators $T_{\alpha}$ are one-to-one and $\left\|T_{\alpha}^{-1}\right\|<1+\varepsilon$. This simple well-known argument is applied, for instance, in [D].

Lemma 2.2 (Another Main Lemma). Let $Y$ be a Banach space, let $E \subset Y^{* *}$ and $F \subset Y^{*}$ be finite-dimensional subspaces, and let $\varepsilon>0$. Then there exists a net $\left(T_{\alpha}\right) \subset \mathcal{F}(E, Y)$ of one-to-one operators satisfying $1^{\circ}-3^{\circ}$ of Corollary 2.1, and $\left\|T_{\alpha}^{-1}\right\|<1+\varepsilon$ for all $\alpha$.

Proof. We first apply the enlarging argument mentioned above. Pick $\delta>0$ such that $(1-2 \delta)^{-1}<1+\varepsilon$. Choose $e_{1}, \ldots, e_{n} \in S_{E}$ to be a $\delta$-net for $S_{E}$. Find $f_{i} \in S_{Y^{*}}$ 
such that $1-\delta<e_{i}\left(f_{i}\right), i=1, \ldots, n$. We may assume, by enlarging $F$ if necessary, that $F$ contains $f_{i}, i=1, \ldots, n$. If $e \in S_{E}$, then clearly $1-2 \delta<\left|e\left(f_{i}\right)\right|$ for some $f_{i}$.

Now let $\left(T_{\alpha}\right)$ be the net in Corollary 2.1. If $e \in S_{E}$, then by $3^{\circ}$,

$$
1-2 \delta<\left|e\left(f_{i}\right)\right|=\left|f_{i}\left(T_{\alpha} e\right)\right| \leq\left\|T_{\alpha} e\right\| .
$$

Hence, $T_{\alpha}$ is one-to-one and $\left\|T_{\alpha}^{-1}\right\| \leq(1-2 \delta)^{-1}<1+\varepsilon$.

The PLR in its improved form due to Johnson, Rosenthal, and Zippin JRZ (see, e.g., [JL, page 53]) is immediate from Lemma 2.2.

Theorem 2.3 (PLR in [JRZ]). Let $Y$ be a Banach space, let $E \subset Y^{* *}$ and $F \subset Y^{*}$ be finite-dimensional subspaces, and let $\varepsilon>0$. Then there exists a one-to-one operator $T \in \mathcal{F}(E, Y)$ such that $\|T\|,\left\|T^{-1}\right\|<1+\varepsilon, T y=y$ for all $y \in E \cap Y$, and $y^{*}\left(T y^{* *}\right)=y^{* *}\left(y^{*}\right)$ for all $y^{* *} \in E$ and $y^{*} \in F$.

2. Let us next apply Lemma 2.2 to obtain the PLR in a more general form due to Behrends [Beh] (see, e.g., [HWW, page 217]).

Theorem 2.4 (PLR in $[\mathrm{Beh}]$ ). Let $Y$ be a Banach space, let $E \subset Y^{* *}, F \subset Y^{*}$ and $H \subset \mathcal{L}(Y, Y)$ be finite-dimensional subspaces, and let $\varepsilon>0$. Denote

$$
E_{H}=E+\operatorname{span}\left\{A^{* *} y^{* *}: A \in H, y^{* *} \in E\right\} \subset Y^{* *} .
$$

Then there exists a one-to-one operator $T: E_{H} \rightarrow Y$ such that

$$
\begin{aligned}
& 1^{\circ}\|T\|,\left\|T^{-1}\right\|<1+\varepsilon, \\
& 2^{\circ} T y=y \text { for all } y \in E_{H} \cap Y, \\
& 3^{\circ} y^{*}\left(T y^{* *}\right)=y^{* *}\left(y^{*}\right) \text { for all } y^{* *} \in E_{H} \text { and } y^{*} \in F, \\
& 4^{\circ}\left\|\left.\left(A T-T A^{* *}\right)\right|_{E}\right\|<\varepsilon \text { for all } A \in S_{H} .
\end{aligned}
$$

Proof. Notice that $E_{H} \subset Y^{* *}$ is a finite-dimensional subspace and apply Lemma 2.2 to $E_{H}, F$, and $\varepsilon$ to find $\left(T_{\alpha}\right) \subset \mathcal{F}\left(E_{H}, Y\right)$. We clearly may assume that $\left\|T_{\alpha}\right\| \leq 2$ for all $\alpha$. Choose $A_{1}, \ldots, A_{n} \in S_{H}$ to be an $\varepsilon / 5$-net for $S_{H}$. Consider now any $A \in\left\{A_{1}, \ldots, A_{n}\right\}$ and observe that

$$
\left.\left(A T_{\alpha}-T_{\alpha} A^{* *}\right)\right|_{E} \rightarrow 0 \text { in the weak operator topology of } \mathcal{L}(E, Y)
$$

because, for all $y^{* *} \in E$ and $y^{*} \in Y^{*}$,

$$
\begin{aligned}
\lim _{\alpha} y^{*}\left(\left(A T_{\alpha}-T_{\alpha} A^{* *}\right) y^{* *}\right) & =\lim _{\alpha} y^{* *}\left(T_{\alpha}^{*} A^{*} y^{*}\right)-\lim _{\alpha} A^{* *} y^{* *}\left(T_{\alpha}^{*} y^{*}\right) \\
& =y^{* *}\left(A^{*} y^{*}\right)-A^{* *} y^{* *}\left(y^{*}\right)=0
\end{aligned}
$$

(since $\left.T_{\alpha}^{*} A^{*} y^{*} \rightarrow A^{*} y^{*}\right|_{E_{H}}$ and $\left.T_{\alpha}^{*} y^{*} \rightarrow y^{*}\right|_{E_{H}}$, and $y^{* *}, A^{* *} y^{* *} \in E_{H}$ ). By passing to convex combinations we may assume that

$$
\left(A T_{\alpha}-T_{\alpha} A^{* *}\right) y^{* *} \rightarrow 0 \quad \forall y^{* *} \in E .
$$

Since this convergence is uniform on the compact set $B_{E}$, we have

$$
\left\|\left.\left(A T_{\alpha}-T_{\alpha} A^{* *}\right)\right|_{E}\right\| \rightarrow 0 .
$$

Thus we may assume that the net $\left(T_{\alpha}\right)$ satisfies

$$
\left\|\left.\left(A_{i} T_{\alpha}-T_{\alpha} A_{i}^{* *}\right)\right|_{E}\right\| \rightarrow 0 \quad \forall i=1, \ldots, n .
$$

It follows that there is some $\alpha$ such that $T:=T_{\alpha}$ satisfies

$$
\left\|\left.\left(A_{i} T-T A_{i}^{* *}\right)\right|_{E}\right\|<\frac{\varepsilon}{5} \quad \forall i=1, \ldots, n .
$$


For this $T$, conditions $1^{\circ}-3^{\circ}$ clearly hold. Also $4^{\circ}$ holds because whenever $A \in S_{H}$ and $\left\|A_{i}-A\right\|<\varepsilon / 5$, then

$$
\begin{aligned}
\left\|\left.\left(A T-T A^{* *}\right)\right|_{E}\right\| & \leq\left\|A_{i}-A\right\|\|T\|+\left\|\left.\left(A_{i} T-T A_{i}^{* *}\right)\right|_{E}\right\|+\|T\|\left\|A_{i}-A\right\| \\
& <2 \frac{\varepsilon}{5}+\frac{\varepsilon}{5}+2 \frac{\varepsilon}{5}=\varepsilon .
\end{aligned}
$$

3. Our last application of Lemma 1.2 tells us that finite-rank operators between dual spaces are "locally conjugate". The following result was obtained by Johnson, Rosenthal, and Zippin in [JRZ, Lemma 3.1 and Corollary 3.2] under the additional restriction that $X$ is finite-dimensional. Their argument would imply also the general result but Lemma 1.2 yields a much quicker proof of it.

Theorem 2.5. Let $X$ and $Y$ be Banach spaces, let $F \subset Y^{*}$ be a finite-dimensional subspace, and let $\varepsilon>0$. If $S \in \mathcal{F}\left(Y^{*}, X^{*}\right)$, then there exists an operator $T \in$ $\mathcal{F}(X, Y)$ such that

$1^{\circ}|\|T\|-\|S\||<\varepsilon$,

$2^{\circ} \operatorname{ran} T^{*}=\operatorname{ran} S$,

$3^{\circ} T^{*} y^{*}=S y^{*}$ for all $y^{*} \in F$,

$4^{\circ} T^{* *} x^{* *}=S^{*} x^{* *}$ for all $x^{* *} \in X^{* *}$ for which $S^{*} x^{* *} \in Y$.

Moreover, if $X=Y$ and $S$ is a projection, then also $T$ can be chosen to be a projection.

Proof. By enlarging $F$ if necessary, we may assume that $S(F)=\operatorname{ran} S$. Since $S^{*} \in \mathcal{F}_{w^{*}}\left(X^{* *}, Y^{* *}\right)$, Lemma 1.2 produces a net $\left(T_{\alpha}\right) \subset \mathcal{F}_{w^{*}}\left(X^{* *}, Y\right)$ such that $\left\|T_{\alpha}\right\| \rightarrow\left\|S^{*}\right\|=\|S\|, T_{\alpha} x^{* *}=S^{*} x^{* *}$ for all $x^{* *} \in X^{* *}$ for which $S^{*} x^{* *} \in Y$, and $T_{\alpha}^{*} y^{*}=S^{* *} y^{*}=S y^{*}$ for all $y^{*} \in F$. Choose $\alpha$ large enough to have $\left|\left\|T_{\alpha}\right\|-\|S\|\right|<$ $\varepsilon$, and put $T=\left.T_{\alpha}\right|_{X}$. Then $T^{* *}=T_{\alpha}$ and thus $1^{\circ}, 4^{\circ}$, and $3^{\circ}$ clearly hold. Also $2^{\circ}$ holds because, by $3^{\circ}$, we have $\operatorname{ran} S=S(F)=T^{*}(F) \subset \operatorname{ran} T^{*}$, and, by $4^{\circ}$, $\operatorname{ker} S^{*} \subset \operatorname{ker} T^{* *}$ and thus $\operatorname{ran} S=\left(\operatorname{ker} S^{*}\right)_{\perp} \supset\left(\operatorname{ker} T^{* *}\right)_{\perp}=\operatorname{ran} T^{*}$.

For the "moreover" part, let $X=Y$ and let $S \in \mathcal{F}\left(X^{*}, X^{*}\right)$ be a projection. By enlarging $F$ if necessary, we may assume that $F \supset \operatorname{ran} S$. By what we have already proven, there exists an operator $T \in \mathcal{F}(X, X)$ satisfying $1^{\circ}-4^{\circ}$. In order to have $T^{2}=T$, it suffices to show that $T^{*} x^{*}=x^{*}$ for all $x^{*} \in \operatorname{ran} T^{*}$. Let $x^{*} \in \operatorname{ran} T^{*}$. Since, by $2^{\circ}, \operatorname{ran} T^{*}=\operatorname{ran} S$, there is $y^{*} \in X^{*}$ such that $x^{*}=S y^{*} \in F$. Thus, by $3^{\circ}, T^{*} x^{*}=T^{*}\left(S y^{*}\right)=S\left(S y^{*}\right)=S y^{*}=x^{*}$.

\section{Generalization of the Main Lemma AND LOCALLY COMPLEMENTED SUBSPACES}

In this final section, we would like to present a general form of our Main Lemma (see Theorem 3.1below) that will make explicit one more reason why the PLR holds! The reason is that, for any Banach space $Y$, there exists an extension operator from $Y^{*}$ to $\left(Y^{* *}\right)^{*}$.

Let $Y$ be a closed subspace of a Banach space $Z$. An operator $\phi \in \mathcal{L}\left(Y^{*}, Z^{*}\right)$ is called an extension operator if $\left(\phi y^{*}\right)(y)=y^{*}(y)$ for all $y^{*} \in Y^{*}$ and all $y \in Y$. If $Y$ is any Banach space, then $Y \subset Y^{* *}$ and, obviously, the canonical embedding $j_{Y^{*}}: Y^{*} \rightarrow Y^{* * *}$ is an extension operator with $\left\|j_{Y^{*}}\right\|=1$.

Pairs of Banach spaces $Z$ and their closed subspaces $Y$ for which there exists an extension operator $\phi \in \mathcal{L}\left(Y^{*}, Z^{*}\right)$ were systematically studied by Fakhoury $[\mathrm{F}]$ and 
Kalton $[\mathrm{K}]$, and various examples are presented in $\mathrm{O}_{2}$, Section 5.5]. Remark that the existence of an extension operator $\phi$ is equivalent to the annihilator $Y^{\perp}$ of $Y$ being complemented in $Z^{*}$. The existence of $\phi$ with $\|\phi\|=1$ means, according to the terminology of Godefroy, Kalton, and Saphar GKS], that $Y$ is an ideal in $Z$. Different subclasses of ideals have been extensively studied by many authors (for references see $\mathrm{O}_{1}$, Section 4]).

Our argument in the proofs of Lemmas 1.1 and 1.2 with some minor modifications (e.g., $Y^{* *}$ has to be replaced by $Z$ and $j_{Y^{*}}$ by $\phi$ ) applies to prove the following generalization of Lemma 1.2 .

Theorem 3.1 (Extended Main Lemma). Let $X$ and $Z$ be Banach spaces, let $Y$ be a closed subspace of $Z$ such that there is an extension operator $\phi \in \mathcal{L}\left(Y^{*}, Z^{*}\right)$, and let $F \subset Y^{*}$ be a finite-dimensional subspace. If $T \in \mathcal{F}_{w^{*}}\left(X^{*}, Z\right)$, then there exists a net $\left(T_{\alpha}\right) \subset \mathcal{F}_{w^{*}}\left(X^{*}, Y\right)$ satisfying

$$
\begin{array}{ll}
1^{\circ} & \left\|T_{\alpha}\right\| \rightarrow\left\|T^{*} \phi\right\|, \\
2^{\circ} & T_{\alpha} x^{*}=T x^{*} \text { for all } x^{*} \in T^{-1}(Y), \\
3^{\circ} & T_{\alpha}^{*} y^{*} \rightarrow T^{*} \phi y^{*} \text { for all } y^{*} \in Y^{*}, \text { in particular, } T_{\alpha}^{*} y^{*}=T^{*} \phi y^{*} \text { for all } y^{*} \in F .
\end{array}
$$

Identifying $\mathcal{F}_{w^{*}}\left(X^{* *}, Z\right)$ and $\mathcal{F}_{w^{*}}\left(X^{* *}, Y\right)$, respectively, with $\mathcal{F}(X, Z)$ and $\mathcal{F}(X, Y)$ via the canonical isometry $\left.T \mapsto T\right|_{X}$ (note that $T^{*}=\left(\left.T\right|_{X}\right)^{*}$ ), Theorem 3.1 immediately yields

Corollary 3.2. Let $X$ and $Z$ be Banach spaces, let $Y$ be a closed subspace of $Z$ such that there is an extension operator $\phi \in \mathcal{L}\left(Y^{*}, Z^{*}\right)$, and let $F \subset Y^{*}$ be a finitedimensional subspace. If $T \in \mathcal{F}(X, Z)$, then there exists a net $\left(T_{\alpha}\right) \subset \mathcal{F}(X, Y)$ satisfying

$1^{\circ}\left\|T_{\alpha}\right\| \rightarrow\left\|T^{*} \phi\right\|$,

$2^{\circ} T_{\alpha} x=T x$ for all $x \in T^{-1}(Y)$,

$3^{\circ} T_{\alpha}^{*} y^{*} \rightarrow T^{*} \phi y^{*}$ for all $y^{*} \in Y^{*}$, in particular, $T_{\alpha}^{*} y^{*}=T^{*} \phi y^{*}$ for all $y^{*} \in F$.

Let us now recall the definition of locally complemented subspaces. A closed subspace $Y$ of a Banach space $Z$ is called locally complemented in $Z$ if there exists a constant $\lambda \geq 1$ such that whenever $E$ is a finite-dimensional subspace of $Z$ and $\varepsilon>0$, there is a linear operator $T: E \rightarrow Y$ with $T x=x$ for all $x \in E \cap Y$ and $\|T\| \leq \lambda+\varepsilon$. In case $\lambda$ works, we say that $Y$ is locally $\lambda$-complemented in $Z$.

The PLR tells us, in particular, that any Banach space $Y$ is locally 1complemented in its bidual $Y^{* *}$. Fakhoury $[\mathrm{F}$ and Kalton $[\mathrm{K}$ independently proved, relying on the PLR, that a closed subspace $Y$ of a Banach space $Z$ is locally complemented whenever there exists an extension operator $\phi \in \mathcal{L}\left(Y^{*}, Z^{*}\right)$. This result is immediate from the special case of Corollary 3.2 when $E \subset Z$ is a finite-dimensional subspace, and $T \in \mathcal{F}(E, Z)$ is the identity embedding.

Corollary 3.3. Let $Z$ be a Banach space and let $Y$ be a closed subspace of $Z$ such that there is an extension operator $\phi \in \mathcal{L}\left(Y^{*}, Z^{*}\right)$. Let $E \subset Z$ and $F \subset Y^{*}$ be finitedimensional subspaces, and let $\varepsilon>0$. Then there is a linear operator $T: E \rightarrow Y$ such that $T y=y$ for all $y \in E \cap Y, y^{*}(T z)=\phi y^{*}(z)$ for all $z \in E$ and $y^{*} \in F$, and $\|T\| \leq\|\phi\|+\varepsilon$. In particular, $Y$ is locally $\|\phi\|$-complemented in $Z$.

Remark 3.1. In Corollary 3.3, one can guarantee that $T$ is one-to-one and $\left\|T^{-1}\right\|<$ $1+\varepsilon$ (by choosing $F$ to be large enough, as in the proof of Lemma 2.2). 
Remark 3.2. In fact, $Y$ is locally complemented in $Z$ if and only if there exists an extension operator $\phi \in \mathcal{L}\left(Y^{*}, Z^{*}\right)$ (see [ $\mathrm{F}$, Theorem 2.14] or [K, Theorem 3.5]). The proof of the "only if" part of this assertion relies on a compactness argument due to Lindenstrauss $[\mathrm{L}$.

\section{ACKNOWLEDGEMENT}

The authors are grateful to the referee for valuable comments and suggestions, which substantially improved the exposition.

\section{REFERENCES}

[Beh] E. Behrends, A generalization of the principle of local reflexivity, Rev. Roumaine Math. Pures Appl. 31 (1986), 293-296. MR0854826 (88a:46011)

[Ber] S.J. Bernau, A unified approach to the principle of local reflexivity, In: H.E. Lacey (ed.), Notes in Banach spaces, 427-439, Univ. Texas Press, Austin, Tex., 1980. MR0606228 (82e:46019)

[C] P.G. Casazza, Approximation properties, In: W.B. Johnson and J. Lindenstrauss (eds.), Handbook of the Geometry of Banach Spaces. Volume 1, 271-316, Elsevier, 2001. MR 1863695 (2003f:46012)

[Ca] J.M.F. Castillo, review of Martinez-Abejón, Antonio. An elementary proof of the principle of local reflexivity. Proc. Amer. Math. Soc. 127 (1999), no. 5, 1397-1398. MR1476378(99h:46008)

[D] D.W. DEAn, The equation $L\left(E, X^{* *}\right)=L(E, X)^{* *}$ and the principle of local reflexivity, Proc. Amer. Math. Soc. 40 (1973), 146-148. MR0324383 (48:2735)

[DU] J. Diestel And J.J. Uhl, Vector Measures, Mathematical Surveys 15. American Mathematical Society, Providence, Rhode Island, 1977. MR0453964(56:12216)

[DSch] N. Dunford And J.T. Schwartz, Linear Operators. Part 1: General Theory, Wiley, New York, 1958. MR1009162 (90g:47001a)

[F] H. Fakhoury, Sélections linéaires associées au théorème de Hahn-Banach, J. Funct. Anal. 11 (1972), 436-452. MR0348457 (50:955)

[GKS] G. Godefroy, N. J. Kalton, And P. D. SAPHAR, Unconditional ideals in Banach spaces, Studia Math. 104 (1993), 13-59. MR.1208038 (94k:46024)

[G] A. Grothendieck, Produits tensoriels topologiques et espaces nucléaires, Mem. Amer. Math. Soc. 16 (1955). MR0075539(17:763c)

[HWW] P. Harmand, D. Werner, AND W. Werner, $M$-ideals in Banach Spaces and Banach Algebras, Lecture Notes in Math., vol. 1547, Springer, Berlin-Heidelberg-New York, 1993. MR.1238713 (94k:46022)

[JL] W.B. JohnSON AND J. Lindenstrauss, Basic concepts in the geometry of Banach spaces, In: W.B. Johnson and J. Lindenstrauss (eds.), Handbook of the Geometry of Banach Spaces. Volume 1, 1-84, Elsevier, 2001. MR.1863689 (2003f:46013)

[JRZ] W.B. Johnson, H.P. Rosenthal, and M. ZipPin, On bases, finite dimensional decompositions and weaker structures in Banach spaces, Israel J. Math. 9 (1971), 488-506. MR0280983 (43:6702)

[K] N.J. Kalton, Locally complemented subspaces and $\mathcal{L}_{p}$-spaces for $0<p<1$, Math. Nachr. 115 (1984), 71-97. MR0755269 (86h:46006)

[L] J. Lindenstrauss, Extension of compact operators, Mem. Amer. Math. Soc. 48 (1964). MR0179580(31:3828)

[LR] J. Lindenstrauss And H.P. Rosenthal, The $\mathcal{L}_{p}$ spaces, Israel J. Math. 7 (1969), 325349. MR0270119 (42:5012)

[M-A] A. Martínez-AbEjón, An elementary proof of the principle of local reflexivity, Proc. Amer. Math. Soc. 127 (1999), 1397-1398. MR1476378 (99h:46008)

$\left[\mathrm{O}_{1}\right]$ E. OJA, Geometry of Banach spaces having shrinking approximations of the identity, Trans. Amer. Math. Soc. 352 (2000), 2801-2823. MR.1675226 (2000j:46034)

$\left[\mathrm{O}_{2}\right]$ E. OJA, Operators that are nuclear whenever they are nuclear for a larger range space, Proc. Edinburgh Math. Soc. 47 (2004), 679-694. MR2097268 (2005g:46040) 
[R] R.A. Ryan, Introduction to Tensor Products of Banach Spaces, Springer, London-BerlinHeidelberg, 2002. MR1888309 (2003f:46030)

[S] Ch. Stegall, A proof of the principle of local reflexivity, Proc. Amer. Math. Soc. 78 (1980), 154-156. MR.0548105 (81e:46012)

Institute of Pure Mathematics, Tartu University, J. Liivi 2, 50409 Tartu, Estonia

E-mail address: eve.oja@ut.ee

Institute of Pure Mathematics, Tartu University, J. Liivi 2, 50409 Tartu, Estonia

E-mail address: mart.poldvere@ut.ee 